\title{
Experimental demonstration of the enhanced transmission through circular and rectangular sub-wavelength apertures using omega-like split-ring resonators
}

\author{
Damla Ates ${ }^{\mathrm{a}, \mathrm{b}, *}$, Filiberto Bilotti $^{\mathrm{c}}$, Alessandro Toscano ${ }^{\mathrm{c}}$, Ekmel Ozbay ${ }^{\mathrm{a}, \mathrm{b}}$ \\ ${ }^{a}$ Department of Electrical and Electronics Engineering, Nanotechnology Research Center, Bilkent University, O6800 Ankara, Turkey \\ ${ }^{\mathrm{b}}$ Department of Physics, Bilkent University, 06800 Ankara, Turkey \\ c "Roma Tre" University, Via della Vasca Navale, 84, 00146 Rome, Italy
}

Received 22 March 2012; received in revised form 5 August 2012; accepted 27 August 2012

Available online 20 September 2012

\begin{abstract}
Enhanced transmission through circular and rectangular sub-wavelength apertures using omega-shaped split-ring resonator is numerically and experimentally demonstrated at microwave frequencies. We report a more than 150,000-fold enhancement through a deep sub-wavelength aperture drilled in a metallic screen. To the authors' best knowledge, this is the highest experimentally obtained enhancement factor reported in the literature. In the paper, we address also the origins and the physical reasons behind the enhancement results. Moreover, we report on the differences occurring when using circular, rectangular apertures as well as doublesided and single-sided omega-like split ring resonator structures.
\end{abstract}

(C) 2012 Elsevier B.V. All rights reserved.

Keywords: Electromagnetic wave transmission; Metamaterials; Resonators

\section{Introduction}

Recently, electromagnetic transmission through subwavelength apertures is receiving a growing interest in the scientific community, due to its potential applications in different scientific fields. Power transmission through electrically small apertures drilled in an opaque screen (e.g. a metallic screen at microwave frequencies) can be controlled in both intensity and frequency by properly designing the geometry of the aperture. Bethe has reported the relation between the transmission and the

\footnotetext{
* Corresponding author at: Department of Electrical and Electronics Engineering, Nanotechnology Research Center, Bilkent University, 06800 Ankara, Turkey.

E-mail address: damla@ee.bilkent.edu.tr (D. Ates).
}

linear dimension $(r)$ of the aperture compared to, the wavelength $(\lambda)$ of the electromagnetic field impinging on the screen as $(r / \lambda)^{4}[1]$. This means that, as expected from intuition, power transmission is extremely low when the apertures are characterized by deeply sub-wavelength dimensions. However, in several applications in science, such as high-capacity optical memories, high-resolution laser lithography, high-capacity optical switches, highefficient and electrically small microwave aperture antennas, high-resolution microwave and optical imaging and screening systems, it is needed to extract even more power from electrically small apertures. In order to get the required transmission enhancement, different physical phenomena can be used.

The experiments have been conducted at optical frequencies, where researchers investigated the effects 
on the transmission efficiency of surface plasmon polaritons excited on the metallic surface of the screens. Particularly, it has been demonstrated that the transmission through sub-wavelength apertures can be enhanced by using the surface plasmon polariton phenomenon $[3,4]$.

Perturbing surface plasmon propagating on a metallic screen by the means of corrugations with proper periodicity surrounding the aperture $[5,6]$, in fact, it is possible to couple the impinging electromagnetic wave with the surface plasmon polaritons and excite the aperture [7]. After Pendry and his colleagues have shown that surface plasmons not only exist in the optical regime but can also exist as spoof plasmons at microwave frequencies, enhanced transmission by periodic corrugations has been demonstrated also in the microwave regime $[8,9]$.

When, metamaterials came into play, it has been demonstrated that enhanced transmission at microwave frequencies can be obtained by replacing corrugations around the aperture with proper metamaterial covers made of epsilon-near-zero or mu-near-zero materials $[10,11]$. A similar but more compact setup in terms of cover thickness has been also demonstrated, by using conjugate-matched layers of epsilon-negative and munegative metamaterials [12].

However, both the corrugation-based approach and the metamaterial approach, rely on the coupling between the impinging waves and propagating surface mode. This leads to an electrically large transverse extension of either corrugations or the cover around the aperture $[13,14]$.

In order to overcome this limitation, another approach to the enhanced transmission has been presented by Aydin et al. and is based on the employment of a single ring resonator placed in front of the aperture $[15,16]$. Since the dimensions of the split ring resonator are comparable to the ones of the aperture, this setup is extremely compact. The experimentally achieved transmission at microwave frequencies demonstrated a 740-fold enhancement factor. Recently, Ates et al. have demonstrated that the transmission enhancement can be increase up to 70,000 -fold (with the same dimensions of the screen and the same free-space measurement setup) by inserting connected split-ring resonators across the aperture [17]. The dramatic increase of the enhancement factor compared to the previous setup was due to: (a) the employment of a symmetric structure (i.e. one split-ring resonator at either side of the screen) and (b) the physical connection between the two split-ring resonators.
In this paper, we report further increased enhancement factors by using a different type of metamaterial resonator, such as the design of omega-like split-ring resonator. The design of omega particles has been first introduced by Saadoum and Engheta in 1992 in order to synthesize bi-anisotropic and "pseudo-chiral" artificial materials and then deeply investigated also by Tretyakov, Simovski and Sochova [18,19]. Recently, it has been demonstrated that omega-like inclusions can be successfully used to obtain also left-handed metamaterials. In this case, in order to eliminate the intrinsic chirality of the medium, two omega-like resonators are deposited in opposite directions on the two sides of a printed circuit board, leading to a low, moderate bandwidth left-handed material design $[20,21]$. Aydin et al. have also reported on the characteristics of such omega-like structures experimentally and numerically [22].

The advantages of using omega-like split-ring resonators instead of conventional split-ring resonators encouraged us to use such structures in the transmission enhancement experiments, in order to obtain better enhancement factors with respect to other reported results [15-17].

\section{Omega-like split-ring resonator configurations}

We connect together two omega shaped split-ring resonators through their arms and we obtain the particle depicted in Fig. 1(a). The samples are produced by printing two identical particles in opposite directions on the two sides of a $0.5 \mathrm{~mm}$ thick FR-4 dielectric board with $0.035 \mathrm{~mm}$ copper thickness as in Fig. 1(a) and (b). The dimensions of the geometrical parameters of the omega samples and metallic screen (see Fig. 1(a)) are $r=3 \mathrm{~mm}, w=1 \mathrm{~mm}, h=7.5 \mathrm{~mm}$ and $l=4 \mathrm{~mm}$.

\section{Experimental environment}

In the experiments, two large copper screens with dimensions $700 \mathrm{~mm} \times 700 \mathrm{~mm} \times 0.5 \mathrm{~mm}$ were used. The first metallic screen had a circular aperture in the center with a radius of $3.75 \mathrm{~mm}$, whereas the second metallic screen had a rectangular aperture with dimensions $3 \mathrm{~mm} \times 7.5 \mathrm{~mm}$. The omega samples are inserted across the apertures as shown in Fig. 1(c) and (d), respectively.

Transmission measurements have been conducted in free-space illuminating the screen by using the conventional waveguide antennas operating in the 
(a)

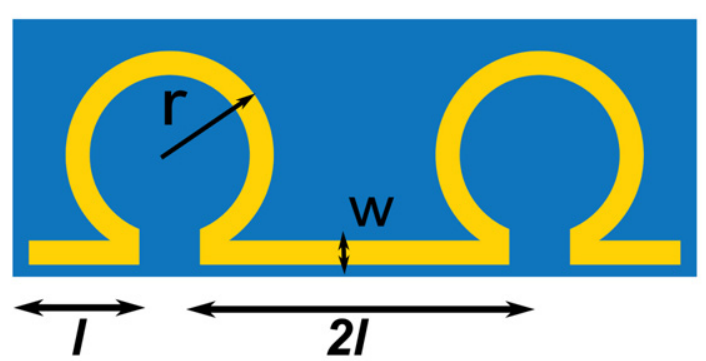

(c)

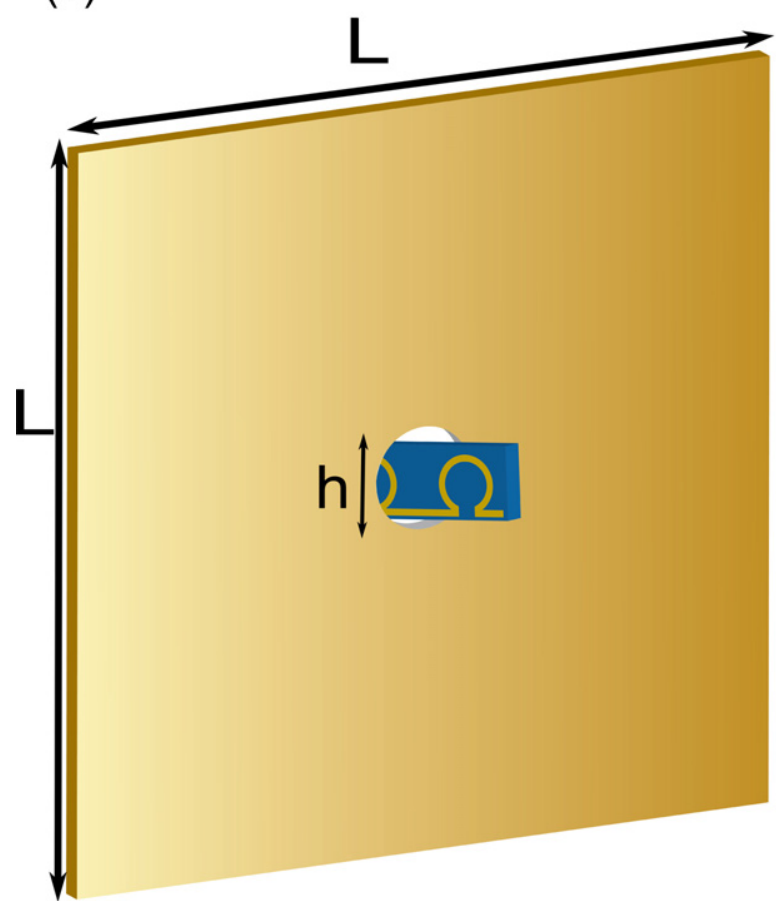

(b)

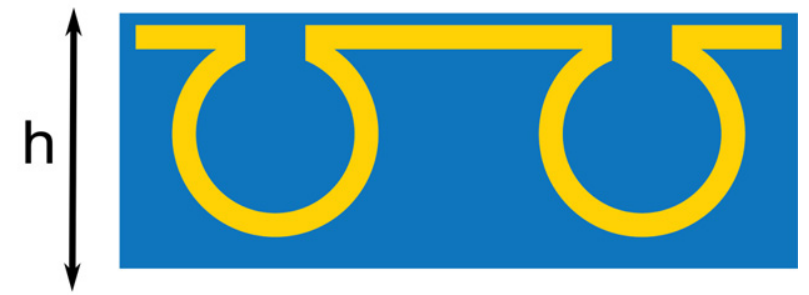

(d)

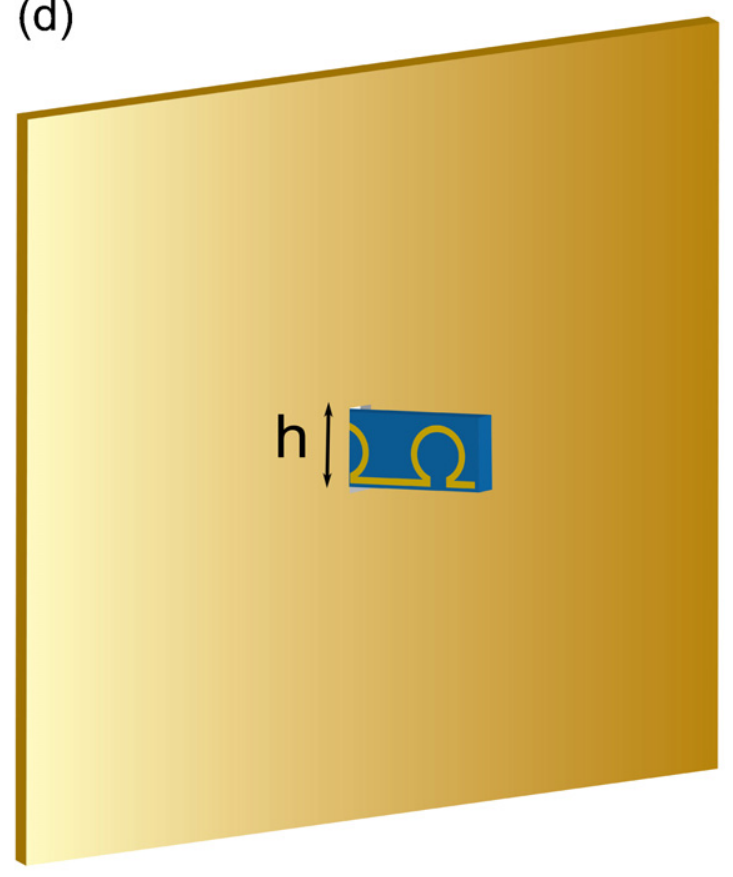

Fig. 1. (a) Schematic of the designed omega-like split-ring resonators from the front side where the parameters are $r=3 \mathrm{~mm}, w=1 \mathrm{~mm}$, and $l=4 \mathrm{~mm}$. (b) Designed omega-like split-ring resonator from the back side. (c) Copper screen with omega-like split-ring resonators inserted in the circular aperture where the dimensions are $h=7.5 \mathrm{~mm}$ and $L=700 \mathrm{~mm}$. (d) Copper screen with omega-like split-ring resonators inserted in the rectangular aperture.

range 3-6 GHz. The metallic screens were placed, one by one, on a holder at the center between the two antennas as depicted in Fig. 2. The waveguide ports were connected to an HP 8510C network analyzer and the S21 transmission parameters were measured.

\section{Transmission enhancement results}

We perform the first transmission measurement with the copper screen having the circular aperture. Then, we performed the second transmission measurement by inserting the omega-like split-ring resonators across the aperture. The measurement results are shown in Fig. 3(a).

Then, we have calculated the enhancement factor from the obtained measurement results by dividing the transmission result of the circular aperture with the omega-like split-ring resonators to the transmission results of the circular aperture alone (shown in Fig. 4(a)). From these results we can see two 


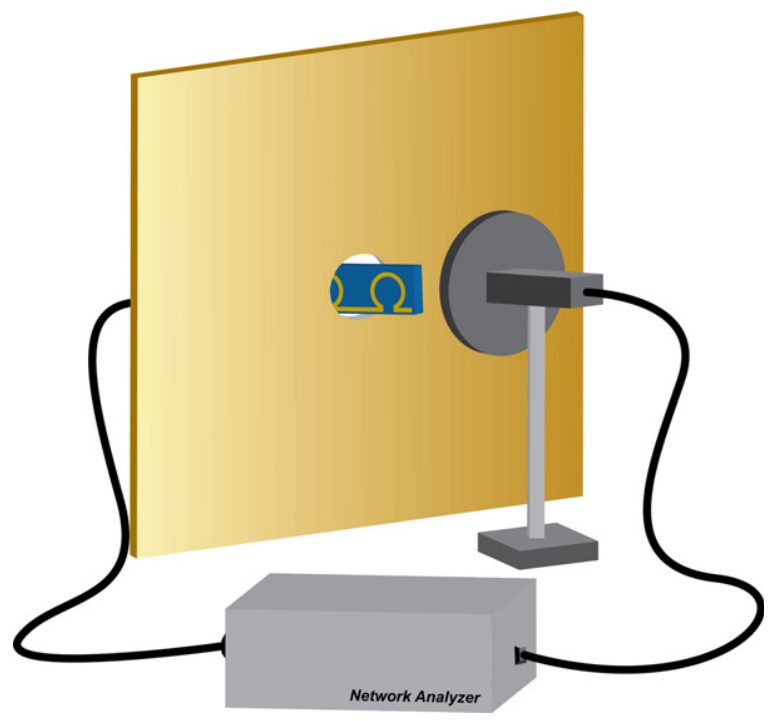

Fig. 2. Schematic demonstration of the transmission enhancement experiments using open-ended waveguide antennas and the HP8510C network analyzer.

enhancement peaks, one at $3.15 \mathrm{GHz}$ and another one at 3.95 GHz. We obtained 4014-fold enhancement at the first peak, whereas at the second peak, we obtained 9262-fold enhancement.

(a)

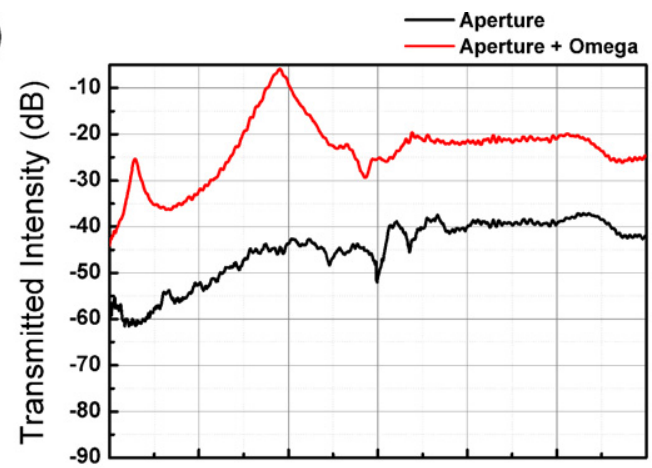

(b)

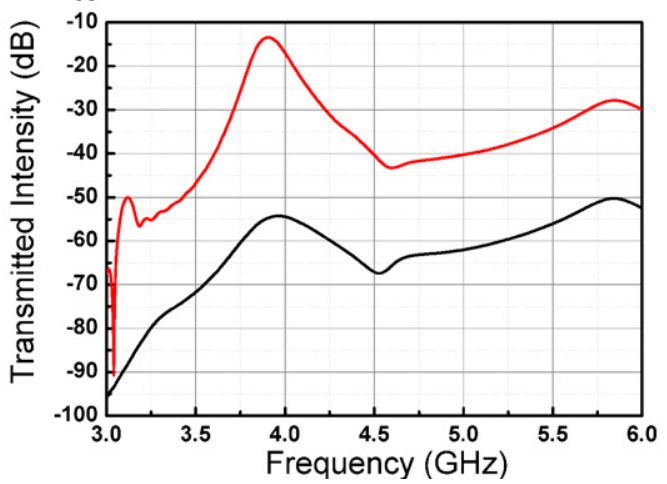

We repeated the measurements by using the metallic screen with the rectangular aperture and the transmission results are depicted in Fig. 3(c).

We have calculated the enhancement factors from the obtained transmission results in a similar way to the enhancement calculations of the circular aperture case. The enhancement result when using the omega shaped split-resonators across the rectangular aperture is presented in Fig. 4(b). We get again two peaks. The first one was located at $3.15 \mathrm{GHz}$, and the second one at $3.95 \mathrm{GHz}$, which are nearly at the same frequencies of transmission peaks of the circular aperture. This means that the transmission frequencies are governed by the geometrical parameters of the omega resonators and are slightly influenced by the surrounding environment. We report a 20,780-fold enhancement at the first peak and a 154,500fold enhancement at the second peak. As already anticipated, these results are much higher than the ones obtained by using different types of resonators [15-17].

\section{Physical analysis and the verification of the experiments by simulations}

The two peaks obtained in both experiments can be explained as follows. The connected omegas are

(c)

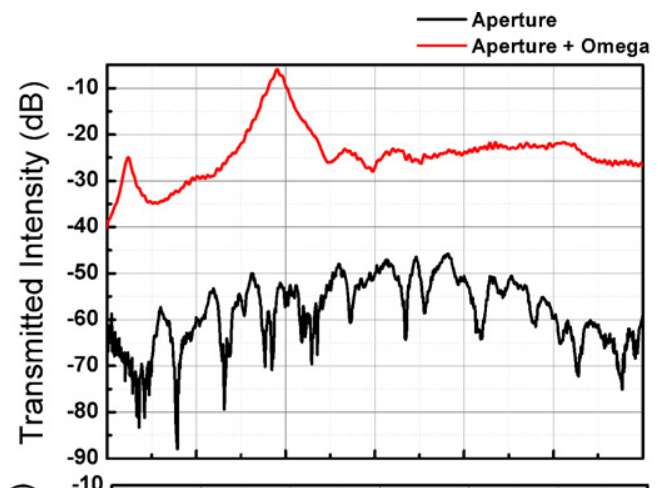

(d)

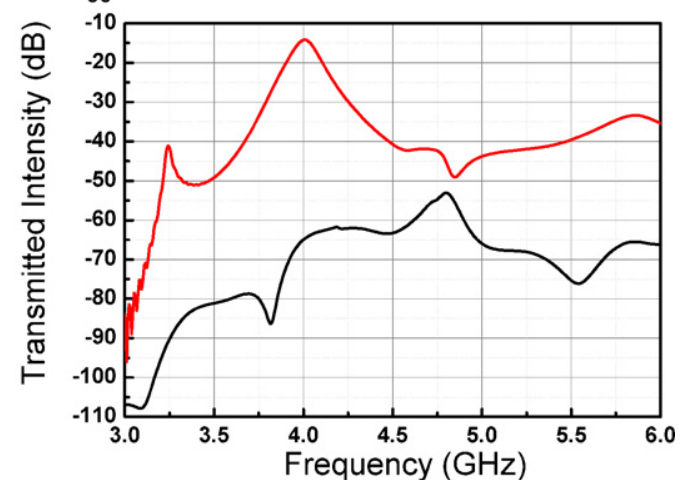

Fig. 3. (a) Measured and (b) simulated results of the transmission intensity (dB) through the circular aperture. (c) Measured and (d) simulated results for the rectangular aperture (solid black line is for the single aperture, solid red line is for the omega-like split-ring resonators inserted across aperture). (For interpretation of the references to color in this figure legend, the reader is referred to the web version of this article.) 
(a)

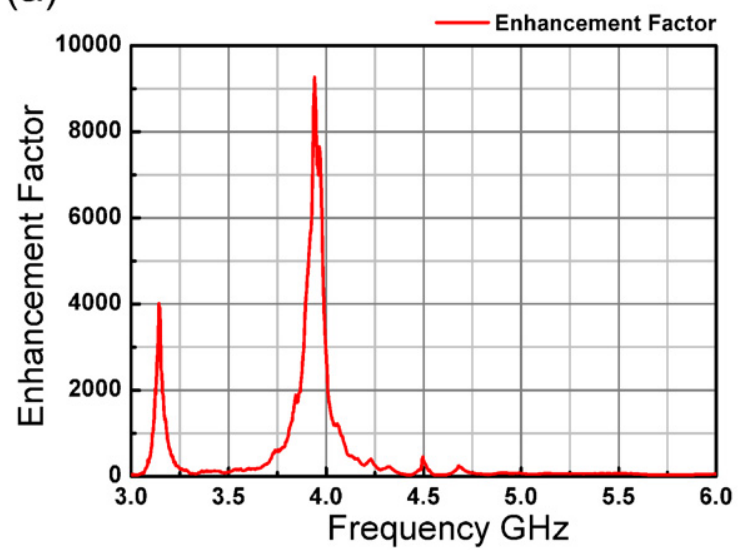

(b)

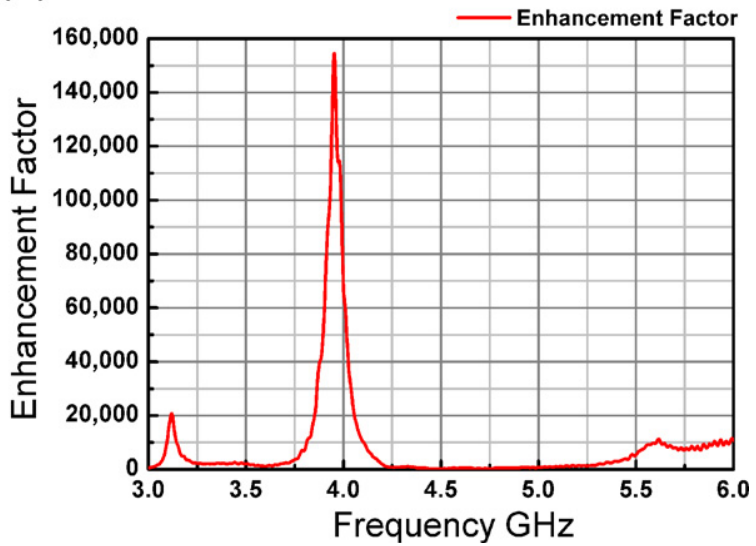

Fig. 4. Enhancement factor in the case of the omega-like split-ring resonators inserted (a) across the circular aperture and (b) across the rectangular aperture.

geometrically symmetric (i.e. made of two identical halves) and, as any symmetric structure, support two fundamental modes of operation. One of them is characterized by an even field symmetry (i.e. the electric field is evenly distributed on the two halves of the structure with respect to the aperture plane) and the other by an odd field symmetry (i.e. the electric field is oddly distributed on the two halves of the structure with respect to the aperture plane). When our designed omega-like split-ring resonators are inserted across the aperture, the loops of the omegas behave as electrically short resonant antennas working in the transmitting mode on the one side of the screen and the receiving mode on the other side of the screen. At the frequency for which the electric field exhibits an even symmetry, the arms of the omegas are not excited; however, the two antennas are electromagnetically coupled through the aperture, as shown in Fig. 5(a).

On the other hand, at the frequency for which the electric field exhibits an odd symmetry as shown in Fig. 5(b), the arms of the omegas are strongly excited and, thus, the two antennas are connected by a transmission line leading to a stronger coupling and, thus, to a higher transmission. In summary, thus, the first peak obtained in the experiments physically originates from the even mode, whereas the second peak from the odd mode. For sake of completeness, we show in Fig. 5(c) the simulated field distribution at a frequency after the two transmission peaks, where the two antennas are not coupled at all and no transmission enhancement is achieved.

To validate the experimental results, we have also performed a numerical analysis of the transmission setup by using CST Microwave Studio. First, we have modeled the two conventional waveguide antennas used in the experiments and we have excited them by waveguide ports. We stayed loyal to the experimental environment as much as possible. Open boundary conditions were employed to emulate the experimental environment. In the simulations, we modeled the FR-4 dielectric slab of the PCB with a relative dielectric constant of $\varepsilon=4$ and a loss tangent of $\delta=0.01$. Simulation results are sketched in Fig. 3(b) for a metallic screen with a circular aperture and in Fig. 3(d) for a metallic screen with a rectangular aperture. The simulation results present a better transmission and higher enhancement factors. One of the main reasons of this discrepancy resides in the diffractions at the edges of the metallic screen, which are cut-off in the simulation environment. The effects of diffractions are explained by Ates et al. [17]. In addition, there are some other minor differences between the simulations and experiments due to manufacturing processes and approximate modeling in the simulation environment.

Furthermore, we wanted to consider the effects of circular and rectangular apertures. As previously anticipated, the two resonant frequencies are independent from the size and geometry of the apertures. The size and geometry of the apertures only influence the transmission intensity and, thus, the enhancement factors. Clearly, the surface area of the rectangular aperture is smaller than the surface area of the circular one. Hence, when using a regular rectangular aperture instead of a regular circular one, the transmission intensity is expected to be lower and, thus, when inserting the omega shaped split-ring resonators, the enhancement factor is expected to be higher. Measured transmission difference between the circular and the 
(a)

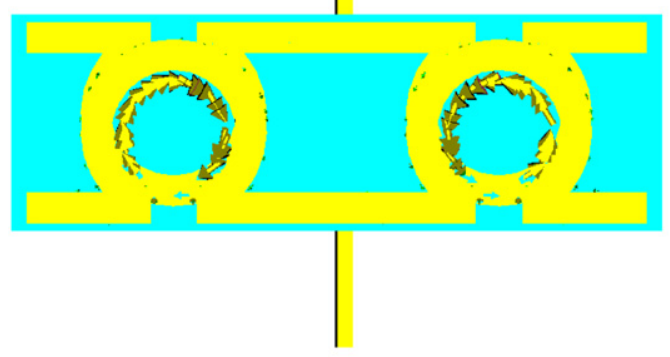

(b)

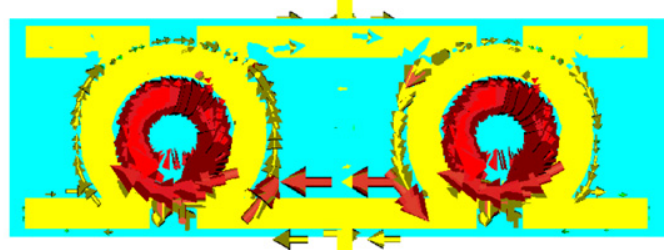

(c)

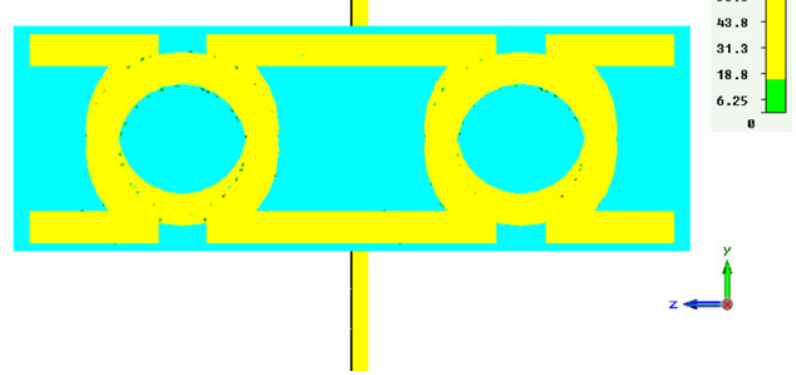

Fig. 5. Surface current density (a) at $3.15 \mathrm{GHz}$ (even mode operation) where the first enhancement peak occurs (MEDIA I), (b) at $3.95 \mathrm{GHz}$ (odd mode operation) where second enhancement peak occurs (MEDIA II), (c) at $5.00 \mathrm{GHz}$ (non-resonant frequency) where no enhancement occurs (MEDIA III).

rectangular aperture is depicted in Fig. 6(a). According to this graph, a circular aperture has higher transmission intensity, due to the size effects. The calculated enhancement factor difference is shown in Fig. 6(b). As explained, by using a rectangular aperture we can obtain 154,500-fold enhancement.

(a)

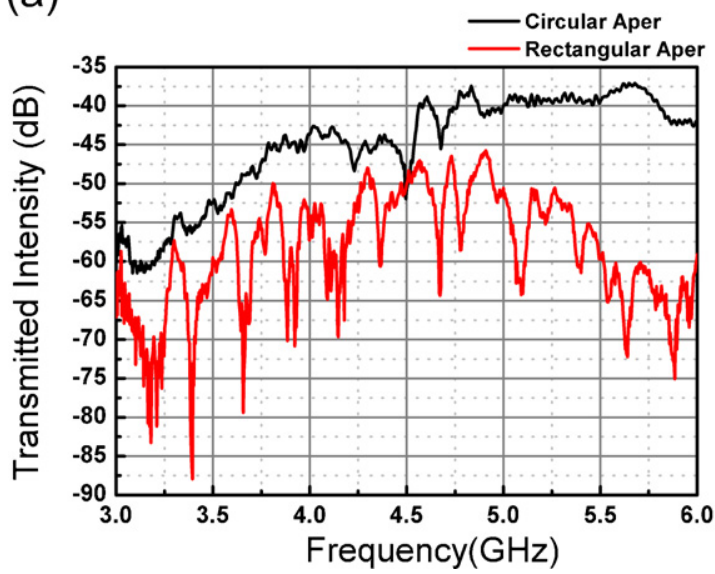

We have also analyzed the effects of the doublesided deposited and single-sided deposited omega-like SRRs. In the first case, we considered the samples that are produced by two identical omega-like SRR samples printed in opposite directions on the two sides of the FR4 board (PCB), which is named as "double-sided

(b)

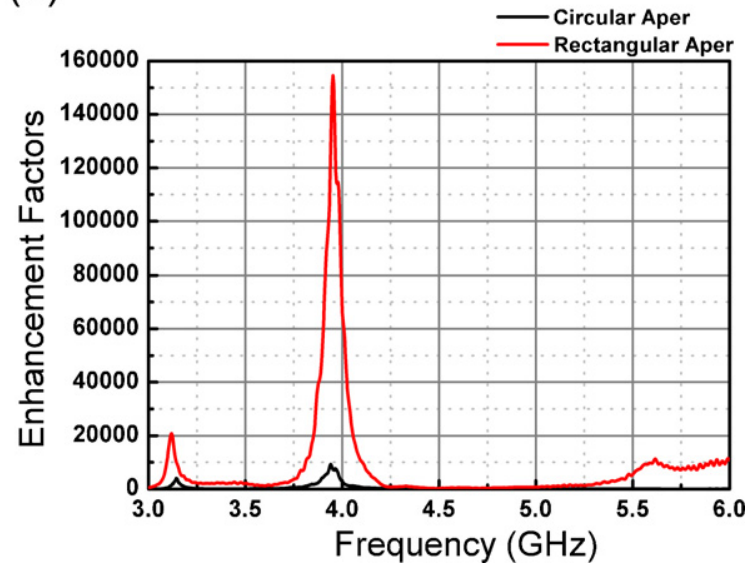

Fig. 6. (a) Difference of the transmission through circular and rectangular apertures and (b) difference of the enhancement factor when omega-like split-ring resonators are inserted across circular and rectangular apertures. 
(a)

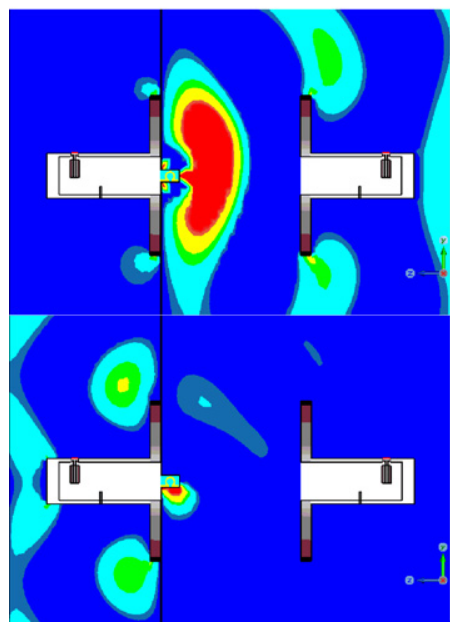

(b)

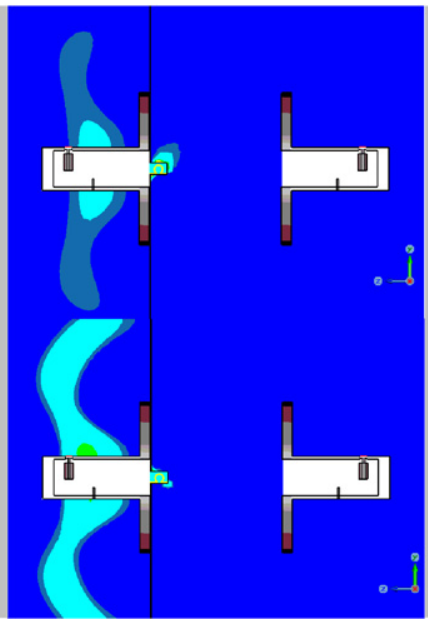

(c)

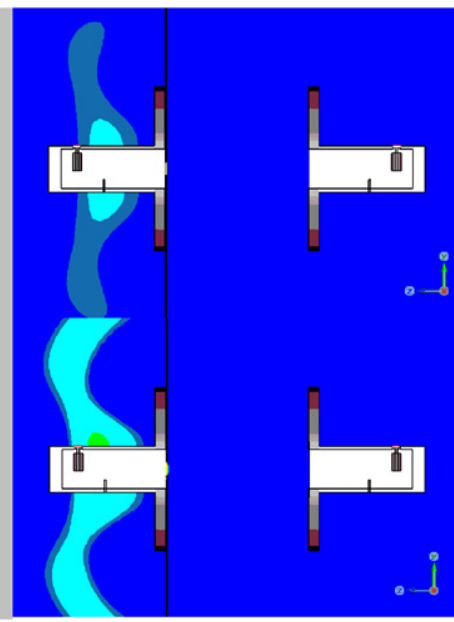

Fig. 7. Electric field distribution maps of the (a) double-sided omega-like SRR across the rectangular aperture at 3.95 GHz (above, MEDIA IV), $5.00 \mathrm{GHz}$ (below, MEDIA V), (b) single-sided omega-like SRR across the rectangular aperture at $3.95 \mathrm{GHz}$ (above, MEDIA VI), 5.00 GHz (below, MEDIA VII), (c) rectangular aperture without omega-like SRR at $3.95 \mathrm{GHz}$ (above, MEDIA VIII), $5.00 \mathrm{GHz}$ (below, MEDIA IX).

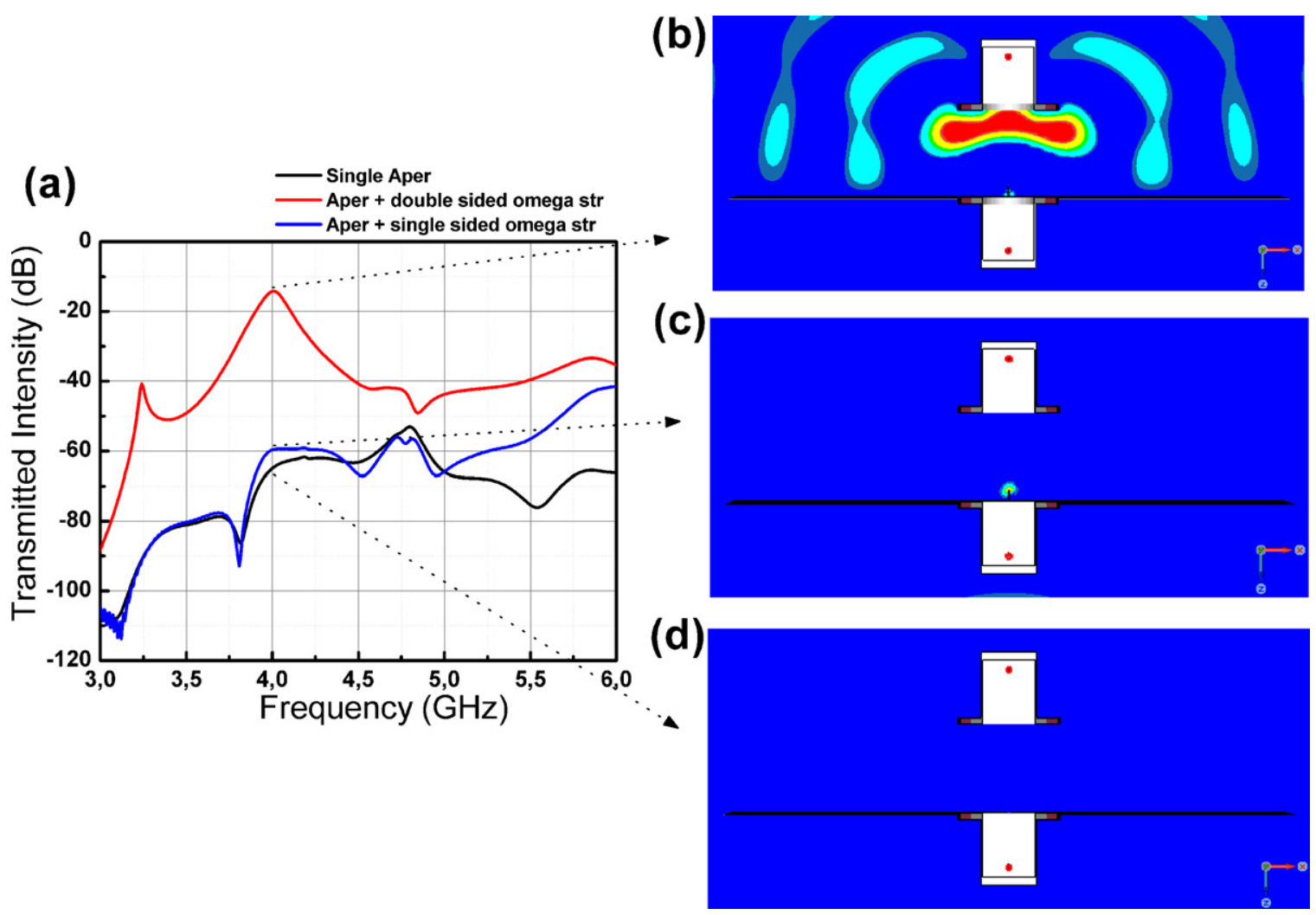

Fig. 8. (a) Transmission characteristics of double-sided (solid red), Single-sided omega-like SRRs (solid blue), aperture without omega-like SRRs (solid black), (b), (c) and (d) electric field distribution maps collected at $3.95 \mathrm{GHz}$ (resonance frequency) respectively. Regarding media: MEDIA X, MEDIA XI and MEDIA XII for (b), (c), and (d) respectively. (For interpretation of the references to color in this figure legend, the reader is referred to the web version of this article.) 
omega-like SRRs". The samples which are named as "single-sided" are produced by single omega-like SRR printed on one of the sides of the PCB. Here, we propose that although the single sided omega structures are still resonators, the reason of the high enhancement is due to the symmetric design of the omega-like SRRs. In order to demonstrate the effect of the symmetric and nonsymmetric design, we have analyzed the electric field distribution maps for three different cases that are shown in Fig. 7. These cases are the field distributions of the double-sided omega-like SRRs inserted in the rectangular aperture (Fig. 7(a)), single-sided omegalike SRR inserted in the rectangular aperture (Fig. 7(b)) and rectangular aperture without omega-like SRRs (Fig. 7(c)) at the frequencies $3.95 \mathrm{GHz}$ (highly resonant frequency where second enhancement peak occurs) and $5.00 \mathrm{GHz}$ (non-resonant frequency where no enhancement occurs).

According to Fig. 7(a), it is observable that the electric field distribution is stronger at the resonant frequency (above) and electric field is weak at the non-resonant frequency (below). Although the field is simulated at the same resonant frequency in Fig. 7(b) (above), the field strength is weaker than the double-sided cases.
This weakness occurs due to non-symmetric nature of the omega-like SRRs design. In other words, the strong enhancement is achieved when the double-sided (symmetric) omega-like SRRs is used. However, the resonant frequency of the single-sided omega is much more sensitive to the surrounding environment than the double-sided one. The resonant frequency, thus, may vary if it is not exactly aligned at the center of the aperture. Nevertheless, the single-sided structure still works as a resonator and the field strength is stronger than the case without a resonator. Finally, the field distributions, which represent weak transmission in the absence of omega-like SRRs, are observed in Fig. 7(c) at $3.95 \mathrm{GHz}$ (above) and $5.00 \mathrm{GHz}$ (below). In this case, there is low transmission and hence no enhancement can be obtained due to absence of the resonator.

A deeper analysis has been done by comparing the transmission spectra of the double-sided, single-sided, and in the absence of omega-like SRR across the rectangular aperture in Fig. 8(a). According to the figure, when the single-sided omega-like SRR is used instead of double-sided, it is apparent that enhancement peaks vanish and transmission behaves as in the case of the absence of omega-like SRRs. Furthermore, we
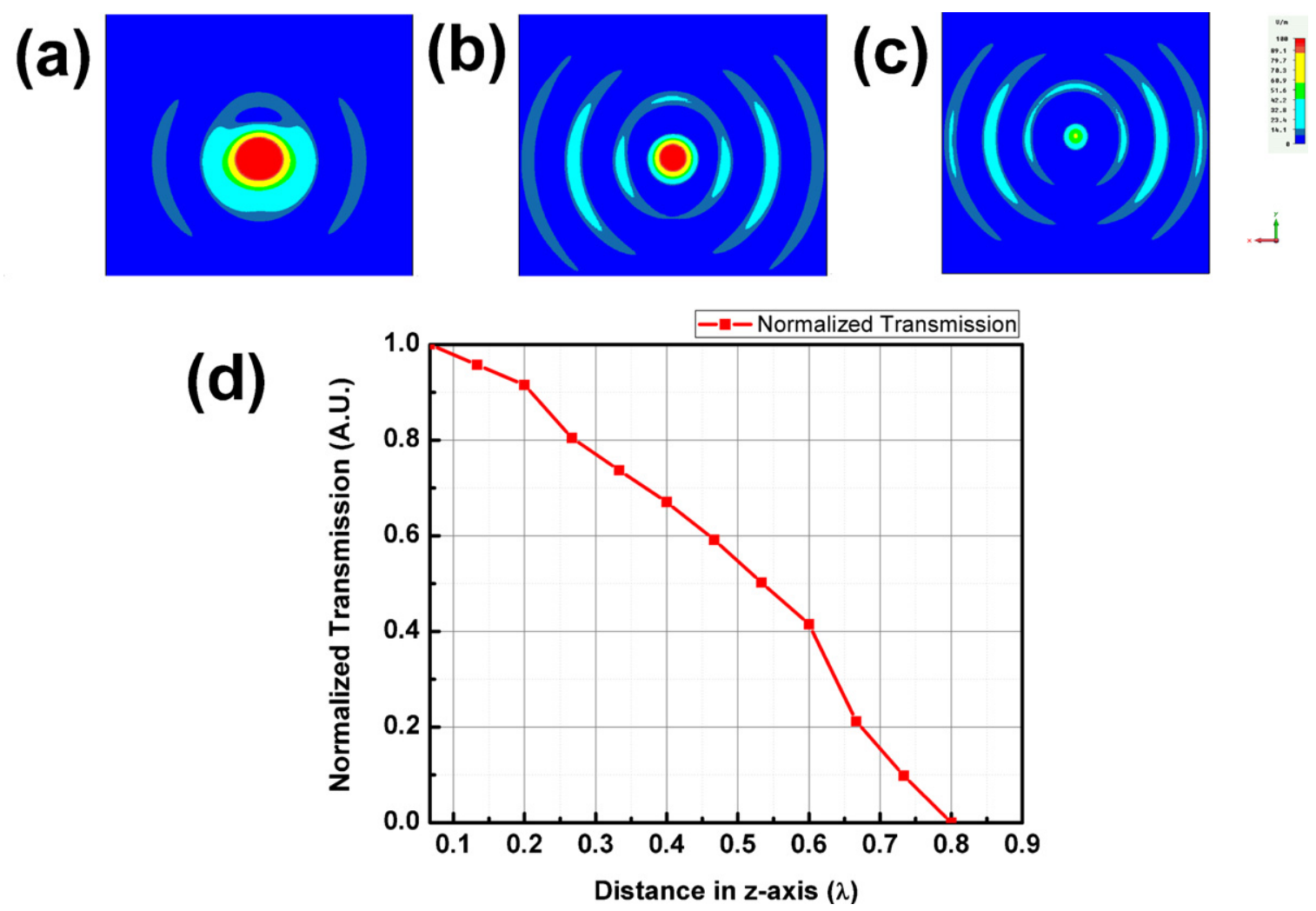

Fig. 9. Field distribution maps (a) $0.1 \lambda$ away (b) $0.5 \lambda$ away, (c) $0.7 \lambda$ away from Omega-like SRR, and (d) normalized transmitted intensity versus distance away from the Omega-like SRR in terms of operational wavelength. 
obtained the field distribution maps for these three cases by field monitors at the highly resonant frequency (3.95 GHz) as in Fig. 8(b)-(d). The strong coupling between the receiver and transmitter compartments occurs due to double-sided omega-like SRR as shown in Fig. 8(b), whereas in Fig. 8(c), coupling is destroyed by using single-sided omega-like SRR. Also, in the absence of omega-like SRRs, there is no coupling; hence no transmission enhancement occurs as presented in Fig. 8(d).

As a final issue, we analyzed the field localization of the transmitted wave at the exit side of the omega-like SRRs. In this case, we have used double-sided omegalike SRRs analyzed at the highly resonant frequency (3.95 GHz). The electric fields at the various distances in the direction of the propagation at the exit side of omega samples are collected by electric field probes. By using these data, we have calculated the normalized electric field intensity. The normalized electric field intensity of the transmitted wave versus distance away from the omega-like SRR in terms of operational wavelength is depicted in Fig. 9(d). In the figure, the transmitted wave decays as the electric field probe moves away from the omega-like SRR at the exit side. Fig. 9(a) represents the field distribution when the probe is $0.1 \lambda$ away from the omega-like SRR, (b) represents when the probe is $0.5 \lambda$ away from the omega-like SRR and finally (c) represents when the probe is $0.7 \lambda$ away from the omega-like SRR. This explains the evanescent field is highly localized near the omega-like SRR and as the probe moves away from the resonator, the evanescent field decays which result in low transmission.

\section{Conclusions and future work}

To summarize, we expected higher transmission enhancement results than the ones already reported in the literature by using regular split-ring resonators, due to the low-loss and bi-anisotropic nature of the proposed omega-like split-ring resonator. The extremely high results, on the order of 150,000-fold, demonstrated both experimentally and numerically, confirm our expectations. We also observed that the geometrical parameters of the omegas control the frequencies at which enhanced transmission is obtained and that such frequencies do not depend on the environment surrounding the resonators. At the final stage, we analyzed the physical origins of the enhancement and the effects of using symmetric and non-symmetric omega-like SRR designs. Furthermore, we demonstrated the highly localized electric fields in the vicinity of omega-like SRRs. The proposed structure can be successfully used to design microwave waveguide components, sensors, electrically small aperture antennas, high-sensitive probes for imaging and screening systems.

\section{Acknowledgements}

This work is supported by projects DPT-HAMIT, EU-PHOME, EU-N4E, NATO-SET-181, and TUBITAK under project Nos., 107A004, 107A012, and 109E301. One of the authors (E.O.) also acknowledges partial support from the Turkish Academy of Sciences.

\section{Appendix A. Supplementary data}

Supplementary data associated with this article can be found, in the online version, at http://dx.doi.org/ 10.1016/j.photonics.2012.08.002.

\section{References}

[1] H.A. Bethe, Theory of diffraction by small holes, Physical Review 66 (1944) 163-182.

[3] G.Q.J.A. Porto, F.J. Garcia-Vidal, J.B. Pendry, Transmission resonances on metallic gratings with very narrow slits, Physical Review Letters 83 (1999) 2845-2848.

[4] L. Martin-Moreno, F.J. Garcia-Vidal, H.J. Lezec, K.M. Pellerin, T. Thio, J.B. Pendry, T.W. Ebbesen, Theory of extraordinary optical transmission through subwavelength hole arrays, Physical Review Letters 86 (2001) 1114-1117.

[5] L. Martin-Moreno, F.J. Garcia-Vidal, H.J. Lezec, A. Degiron, T.W. Ebbesen, Physical Review Letters 90 (2003) 167401.

[6] S. Akarca-Biyikli, I. Bulu, E. Ozbay, Enhanced transmission of microwave radiation in one-dimensional metallic gratings with subwavelength aperture, Applied Physics Letters 85 (2004) 1098-1100.

[7] S. Akarca-Biyikli, I. Bulu, E. Ozbay, Resonant excitation of surface plasmons in one-dimensional metallic grating structures at microwave frequencies, Journal of Optics A: Pure and Applied Optics 7 (2005) S159-S164.

[8] J. Pendry, L. Martin-Moreno, F. Garcia-Vidal, Mimicking surface plasmons with structured surfaces, Science 305 (2004) 847-848.

[9] E. Ozbay, Plasmonics: merging photonics and electronics at nanoscale dimensions, Science 311 (2006) 189.

[10] N. Katsarakis, M. Kafesaki, I. Tsiapa, E.N. Economou, C.M. Soukoulis, High transmittance left-handed materials involving symmetrics split-ring resonators, Photonics and Nanostructures - Fundamentals and Applications 5 (2007) 149-155.

[11] A. Alu, F. Bilotti, N. Engheta, L. Vegni, Metamaterial covers over a small aperture, IEEE Transactions on Antennas Propagation 54 (2006) 1632-1643.

[12] A. Alù, F. Bilotti, N. Engheta, L. Vegni, Subwavelength planer leaky-wave components with metamaterial bilayers, IEEE Transactions on Antennas Propagation 55 (2007) 882-891.

[13] R. Marques, F. Mesa, J. Martes, F. Medina, Comparative analysis of edge- and broadside-coupled split ring resonator for meta- 
material design-theory and experiments, IEEE Transactions on Antennas Propagation 51 (2003) 2572-2581.

[14] F. Bilotti, L. Scorrano, E. Ozbay, L. Vegni, Enhanced transmission through a sub-wavelength aperture: resonant approaches employing metamaterials, Journal of Optics A: Pure and Applied Optics 11 (2009) 114029.

[15] K. Aydin, A.O. Cakmak, L. Sahin, Z. Li, F. Bilotti, L. Vegni, E. Ozbay, Split-ring-resonator-coupled enhanced transmission through a single subwavelength aperture, Physical Review Letters 102 (2009) 013904.

[16] A.O. Cakmak, K. Aydin, E. Colak, F. Bilotti, L. Vegni, E. Ozbay, Enhanced transmission through a subwavelength aperture using metamaterials, Applied Physics Letters 95 (2009) 052103.

[17] D. Ates, A.O. Cakmak, E. Colak, R. Zhao, C.M. Soukoulis, E. Ozbay, Transmission enhancement through deep subwavelength apertures using connected split ring resonators, Optics Express 18 (2010) 3952-3966.

[18] M.M.I. Saadoum, N. Engheta, A reciprocal phase shifter using novel pseudochiral or $\omega$ medium, Microwave and Optical Technology Letters 5 (1992) 184

[19] C.R. Simovski, S.A. Tretyakov, A.A. Sochava, B. Sauviac, F. Mariotte, T.G. Kharina, Antenna model for conductive omega particles, Journal of Electromagnetic Waves and Applications 11 (1997) 1509-1530.

[20] C.R. Simovski, S. He, Frequency range and explicit expressions for negative permittivity and permeablity of an isotropic medium formed by a lattice of perfectly conducting Omega-particles, Physical Review A 311 (2003) 254.

[21] L. Ran, J. Huangfu, H. Chen, Y. Li, X. Zhang, K. Chen, J.A Kong, Microwave solid-state left-handed material with a broad bandwidth and an ultralow loss, Physical Review B 70 (2004) 073102.

[22] K. Aydin, Z. Li, S. Bilge, E. Ozbay, Experimental and numerical study of omega type bianisotropic metamaterial combined with a negative permittivity medium, Photonics and Nanostructures Fundamentals and Applications 6 (2008) 116-121.

\section{Further reading}

[2] T.W. Ebbesen, H.J. Lezec, H.F. Ghaemi, T. Thio, P.A. Wolff, Extraordinary optical transmission through sub-wavelength hole arrays, Nature 391 (1998) 667-669. 